\title{
CORRELATIONS BETWEEN SUPERMASSIVE BLACK HOLES, VELOCITY DISPERSIONS, AND MASS DEFICITS IN ELLIPTICAL GALAXIES WITH CORES*
}

\author{
JOHN KORMENDY ${ }^{1}$ AND RALF BendeR ${ }^{2,3}$ \\ ${ }^{1}$ Department of Astronomy, University of Texas at Austin, 1 University Station C1400, Austin, Texas 78712-0259, USA; kormendy@astro.as.utexas.edu \\ ${ }^{2}$ Universitäts-Sternwarte, Scheinerstrasse 1, München D-81679, Germany \\ ${ }^{3}$ Max-Planck-Institut für Extraterrestrische Physik, Giessenbachstrasse, D-85748 Garching-bei-München, Germany; bender@mpe.mpg.de \\ Received 2008 September 8; accepted 2008 November 24; published 2009 January 19
}

\begin{abstract}
High-dynamic-range surface photometry in a companion paper makes possible accurate measurement of the stellar light deficits $L_{\text {def }}$ and mass deficits $M_{\text {def }}$ associated with the cores of elliptical galaxies. We show that $L_{\text {def }}$ correlates with velocity dispersions $\sigma$ of the host galaxy bulge averaged outside the central region that may be affected by a supermassive black hole $(\mathrm{BH})$. We confirm that $L_{\text {def }}$ correlates with $\mathrm{BH}$ mass $M_{\bullet}$. Also, the fractional light deficit $L_{\text {def }} / L_{*}$ correlates with $M_{\bullet} / M_{*}$, the ratio of BH mass to the galaxy stellar mass. All three correlations have scatter similar to or smaller than the scatter in the well-known correlation between $M_{\bullet}$ and $\sigma$. The new correlations are remarkable in view of the dichotomy between ellipticals with cores and those with central extra light. Core light deficit correlates closely with $M_{\bullet}$ and $\sigma$, but extra light does not. This supports the suggestion that extra light Es are made in wet mergers with starbursts whereas core Es are made in dry mergers. After dry mergers, cores are believed to be scoured by BH binaries that fling stars away as their orbits decay or by BHs that sink back to the center after recoiling from anisotropic gravitational radiation emitted when they merge. Direct evidence for these mechanisms has been elusive. We interpret the new correlations as the "smoking gun" that connects cores with BHs. Together, the $M_{\bullet}-\sigma$ and $M_{\bullet}-L_{\text {def }}$ correlations give us two independent ways to estimate BH masses in core Es.
\end{abstract}

Key words: galaxies: elliptical and lenticular, $\mathrm{cD}$ - galaxies: evolution - galaxies: formation - galaxies: nuclei galaxies: photometry - galaxies: structure

\section{INTRODUCTION}

Research on supermassive black holes (BHs) in galaxy nuclei centers on the growing evidence that $\mathrm{BH}$ growth and galaxy evolution are connected (Ho 2004). The most striking evidence is the discovery of correlations between $\mathrm{BH}$ mass $M_{\bullet}$ and bulge (but not disk) luminosity (Kormendy 1993; Kormendy \& Richstone 1995; Magorrian et al. 1998; Kormendy \& Gebhardt 2001) and between $M_{\bullet}$ and the velocity dispersion $\sigma$ of the bulge outside the region influenced by the BH (Ferrarese \& Merritt 2000; Gebhardt et al. 2000b). These scaling relations are clues to formation physics, but they do not point to specific astrophysical processes. So the literature on their interpretation has been extraordinarily wide ranging.

This Letter reports tight mutual correlations between $M_{\bullet}, \sigma$, and the light deficits $L_{\text {def }}$ that define cores in elliptical galaxies. These point more directly to formation processes. In particular, we find a correlation between the fundamental observables $L_{\mathrm{def}}$ and $\sigma$ that is as tight as the well-known $M_{\bullet}-\sigma$ correlation.

To set the stage, we recall that it is difficult to understand how cores can form in galaxies that are made by major mergers. We define $^{4}$ a core as the region near the center of a bulge or elliptical interior to the radius where the outer, steep brightness profile shows a downward break to a shallow, central profile (Kormendy 1999; Kormendy et al. 2008). Caon et al. (1993) showed that the

\footnotetext{
* Based on observations made with the NASA/ESA Hubble Space Telescope, obtained from the Data Archive at STScI, which is operated by AURA, Inc., under NASA contract NAS 5-26555. These observations are associated with program numbers $5477,7868,8686$, and 9401.

4 This is essentially equivalent to the classical definition that cores resemble the central profiles of nonsingular isothermal spheres or the Nuker team definition that cores are regions near the center where the projected brightness profile shows a shallow cusp, $I(r) \propto r^{-\gamma}$ with $\gamma \leqslant 0.3$, interior to a downward profile break toward the center (Kormendy et al. 1994; Lauer et al. 1995; Faber et al. 1997; see Kormendy et al. 2008 for discussion).
}

outer profiles $I(r)$ are best modeled by Sérsic (1968) functions, $\log I(r) \propto r^{1 / n}$. Many authors confirm this. Kormendy et al. (2008) find that Sérsic functions fit ellipticals with remarkable precision over large dynamic ranges. With respect to these outer Sérsic fits, cores show central light deficits. Why?

The problem is that galaxy mergers tend to preserve the highest progenitor densities. Lower-luminosity galaxies have higher central densities (Kormendy 1985, 1987; Lauer 1985; Faber et al. 1997). When ellipticals or bulges merge, this tends to destroy the above correlation (Faber et al. 1997). A possible solution is that cores may be scoured by the orbital decay of binary BHs that form in galaxy mergers (e. g., Ebisuzaki et al. 1991; Faber et al. 1997; Milosavljević \& Merritt 2001; Milosavljević et al. 2002; Merritt 2006). The orbit shrinks as the binary flings stars away. This decreases the surface brightness and excavates a core. The effect of a series of mergers should be cumulative; if the central mass deficit after one merger is $f M_{\bullet}$, then the mass deficit after $N$ major mergers should be $\sim N f M_{\bullet}$. The above papers predict that $f \sim 0.5$ to 2 . Past observations of mass deficits $M_{\text {def }}$ were roughly consistent with this picture; $M_{\text {def }} \propto M_{\bullet}$ and $N f \sim 1-5$, consistent with formation by several successive dissipationless mergers (Milosavljević \& Merritt 2001; Milosavljević et al. 2002; Ravindranath et al. 2002; Graham 2004; Ferrarese et al. 2006; Merritt 2006).

\section{CORE MASS DEFICIT VERSUS BH MASS}

The key to this Letter is high-accuracy surface photometry of all known ellipticals in the Virgo cluster presented in Kormendy et al. (2008, hereafter KFCB). Composite profiles are measured and assembled from 6-11 sources for each core elliptical. A variety of telescopes including the Hubble Space Telescope (HST) and wide-field, ground-based telescopes provide large dynamic range. Comparison of many data sources minimizes 
systematic errors. KFCB find that Sérsic functions accurately fit $93-99 \%$ of the light of each galaxy. Central departures of the profiles from these fits can be interpreted with confidence. One result is an improved demonstration that profile shape participates in the well-known dichotomy into two kinds of ellipticals (Bender 1988; Bender et al. 1989; Nieto et al. 1991; Kormendy \& Bender 1996). HST photometry shows that ellipticals come in core and coreless varieties (Lauer et al. 1995, 2005, 2007; Gebhardt et al. 1996; Rest et al. 2001; Ravindranath et al. 2001). Faber et al. (1997) show that this distinction correlates with the rest of the dichotomy: core ellipticals generally have boxy distorted isophotes and rotate slowly, whereas coreless ellipticals have disky distorted isophotes and rotate rapidly. KFCB confirm results of Kormendy (1999) that coreless Es do not have featureless "power-law profiles" near their centers but rather show distinct components-extra light above the inward extrapolation of their outer Sérsic profiles. The extra light resembles the extra central components predicted in $n$-body simulations of galaxy mergers in which gas dissipation feeds starbursts (Mihos \& Hernquist 1994; see Hopkins et al. 2008 for extensive simulations). KFCB conclude, in agreement with Faber et al. (1997), that "power-law Es" (now more accurately called "extra light Es") formed in dissipative ("wet") mergers with starbursts, whereas core Es formed in dissipationless ("dry") mergers with binary BH scouring.

KFCB calculate with improved accuracy the mass excesses in extra light and the mass deficits (not) in cores. Figure 1 shows that extra light correlates only weakly with $M_{\text {. }}$. But mass deficits correlate closely with $M_{\text {. }}$. Moreover, the mass deficit is a larger multiple of the $\mathrm{BH}$ mass than previously thought. The mean $\left\langle\log M_{\text {def }} / M_{\bullet}\right\rangle=1.02 \pm 0.07$. These values are surprisingly large in comparison to the prediction (Merritt 2006) that $M_{\text {def }} / M_{\bullet} \simeq 0.5$ per major merger. However, with a more accurate treatment of the late stages of $\mathrm{BH}$ mergers, Merritt et al. (2007) find that $M_{\text {def }} / M_{\bullet}$ can be as large as $\sim 4$ per merger. Second, an additional process has been proposed to make large- $M_{\text {def }} / M_{\text {}}$ cores (Merritt et al. 2004; BoylanKolchin et al. 2004; Gualandris \& Merritt 2008). Coalescing binary BHs emit gravitational radiation anisotropically; they recoil at velocities comparable to galaxy escape velocities. If they do not escape, they decay back to the center by dynamical friction. In the process, they heat the core. Gualandris \& Merritt (2008) estimate that they can excavate as much as $M_{\text {def }} / M_{\bullet} \sim 5$ in addition to the mass that was scoured by the pre-coalescence binary. So our observations present no problem for the idea that cores in giant ellipticals are made by a combination of the above two BH scouring mechanisms acting over the course of one or more successive dry mergers. We do not estimate the number of mergers, because theoretical predictions of $f$ are uncertain and because our measurements of $M_{\text {def }}$ depend on the assumption that unscoured profiles are exactly Sérsic.

The purpose of this Letter is to pursue the correlation in the bottom panel of Figure 1 in more detail. In particular, we "deconstruct" it to correlations between basic observables.

First, an explanation of the measurements is necessary. The amount of extra or missing light is calculated by integrating the two-dimensional brightness distribution of the galaxy from the center to the inner limit $r_{\text {min }}$ of the outer Sérsic function's radial fit range. From this luminosity, we subtract the integral of the fitted Sérsic function over the same radial range, keeping the ellipticity $\epsilon$ of the Sérsic function fixed at $\epsilon\left(r_{\min }\right)$. Internal errors are estimated by substituting plausible extrapolations of the $\epsilon(r)$ profile into the region at $r<r_{\text {min }}$. Our measurements
Table 1

Core and BH Data

\begin{tabular}{lccccc}
\hline \hline Galaxy & $\begin{array}{c}D \\
(\mathrm{Mpc})\end{array}$ & $M_{V T}$ & $M_{V, \text { def }}$ & $\begin{array}{c}\sigma \\
\left(\mathrm{km} \mathrm{s}^{-1}\right)\end{array}$ & $\begin{array}{c}\log M_{\bullet} \\
\left(M_{\odot}\right)\end{array}$ \\
\hline NGC 3379 & 10.57 & -20.99 & $-15.57_{-0.05}^{+0.05}$ & 206 & $8.000_{-0.301}^{+0.204}$ \\
NGC 4261 & 31.60 & -22.60 & $-18.26_{-0.03}^{+0.03}$ & 315 & $8.716_{-0.103}^{+0.076}$ \\
NGC 4365 & 23.33 & -22.63 & $-17.13_{-0.11}^{+0.13}$ & 261 & $8.595_{-0.3}^{+0.3}$ \\
NGC 4374 & 18.45 & -22.63 & $-18.08_{-0.04}^{+0.04}$ & 296 & $8.948_{-0.187}^{+0.187}$ \\
NGC 4382 & 17.86 & -22.54 & $-15.68_{-0.31}^{+0.44}$ & 187 & $8.013_{-0.3}^{+0.3}$ \\
NGC 4406 & 16.83 & -22.69 & $-15.77_{-0.06}^{+0.07}$ & 228 & $8.359_{-0.3}^{+0.3}$ \\
NGC 4472 & 17.14 & -23.24 & $-17.49_{-0.10}^{+0.11}$ & 291 & $8.785_{-0.3}^{+0.3}$ \\
NGC 4486 & 17.22 & -22.95 & $-19.51_{-0.23}^{+0.30}$ & 375 & $9.565_{-0.143}^{+0.108}$ \\
NGC 4552 & 15.85 & -21.66 & $-16.88_{-0.08}^{+0.08}$ & 248 & $8.506_{-0.3}^{+0.3}$ \\
NGC 4636 & 14.66 & -22.10 & $-15.46_{-0.18}^{+0.22}$ & 202 & $8.147_{-0.3}^{+0.3}$ \\
NGC 4649 & 17.30 & -22.75 & $-17.80_{-0.07}^{+0.07}$ & 375 & $9.314_{-0.155}^{+0.079}$ \\
\hline
\end{tabular}

depend critically on the high accuracy and large dynamic range of the composite brightness profiles in KFCB; these constrain Sérsic $n$ values better than previous work. Our values of $n$ are slightly larger than those derived previously (see KFCB), resulting in larger estimates of light deficits. Also, we calculate the difference between the integral of our outer Sérsic fit and the integral of the galaxy profile, not the difference between a Sérsic fit and a core-Sérsic fit as in Ferrarese et al. (2006).

Missing light is converted to missing mass using mass-tolight ratios $M / L_{V} \propto L_{V}^{0.36}$ fitted to the SAURON sample of Cappellari et al. (2006) (zeropoint $M / L_{V}=6.07$ at $M_{V}=$ -21.6 ; estimated error in $\log M / L_{V}=0.153$ ). For galaxies with $\mathrm{BH}$ detections, we take $M / L_{V}$ and $M_{\bullet}$ from the dynamical modeling paper. Otherwise, $M_{\bullet}$ is derived using the $M_{\bullet}-$ $\sigma$ correlation as fitted by Tremaine et al. (2002). Velocity dispersions are mostly from Tremaine et al. (2002); Bender et al. (1994), and Kormendy \& Gebhardt (2001). The sample, total absolute magnitudes $M_{V T}$, and light deficits $M_{V \text {,def }}$ are from KFCB with NGC 3379 added. Distances are from Mei et al. (2007) or Tonry et al. (2001). The data are listed in Table 1.

\section{CORRELATIONS BETWEEN LIGHT DEFICIT AND BH MASS}

Figure 2 shows the fraction of the total $V$-band light of the galaxy that is "missing" in the core versus the ratio of the $\mathrm{BH}$ mass to the stellar mass of the galaxy. As we would expect if $M_{\text {def }} / M_{\bullet} \simeq$ constant (Figure 1), there is good correlation and the slope is consistent with 1 .

The lines in Figures 2-4 are least-squares fits following Tremaine et al. (2002): (i) the dependent and independent variables $x$ and $y$ are treated symmetrically; (ii) we calculate regressions of $y-\bar{y}$ on $x-\bar{x}$ so errors in the fit parameter are essentially uncorrelated and minimized (Tremaine et al. 2002, p. 742); (iii) we calculate a fit using the estimated errors in both parameters and adding intrinsic scatter to bring the reduced $\chi^{2}$ to 1 ; (iv) we calculate a second fit by assigning equal errors to all $x$ and different equal errors to all $y$ and finding the values of those errors that result in a reduced $\chi^{2}$ of 1 , thus providing partial protection against the danger that the assumed errors are not realistic, and (v) we adopt the mean of these fits as was done by Tremaine et al. (2002). In the above, e.g., $\bar{x}$ is the mean of the $x$ values. In Figure 2, the adopted correlation is

$$
\frac{L_{V, \mathrm{def}} / L_{V}}{0.01}=\left(1.066_{-0.193}^{+0.236}\right)\left(\frac{M_{\bullet} / M}{0.001}\right)^{1.123 \pm 0.235},
$$




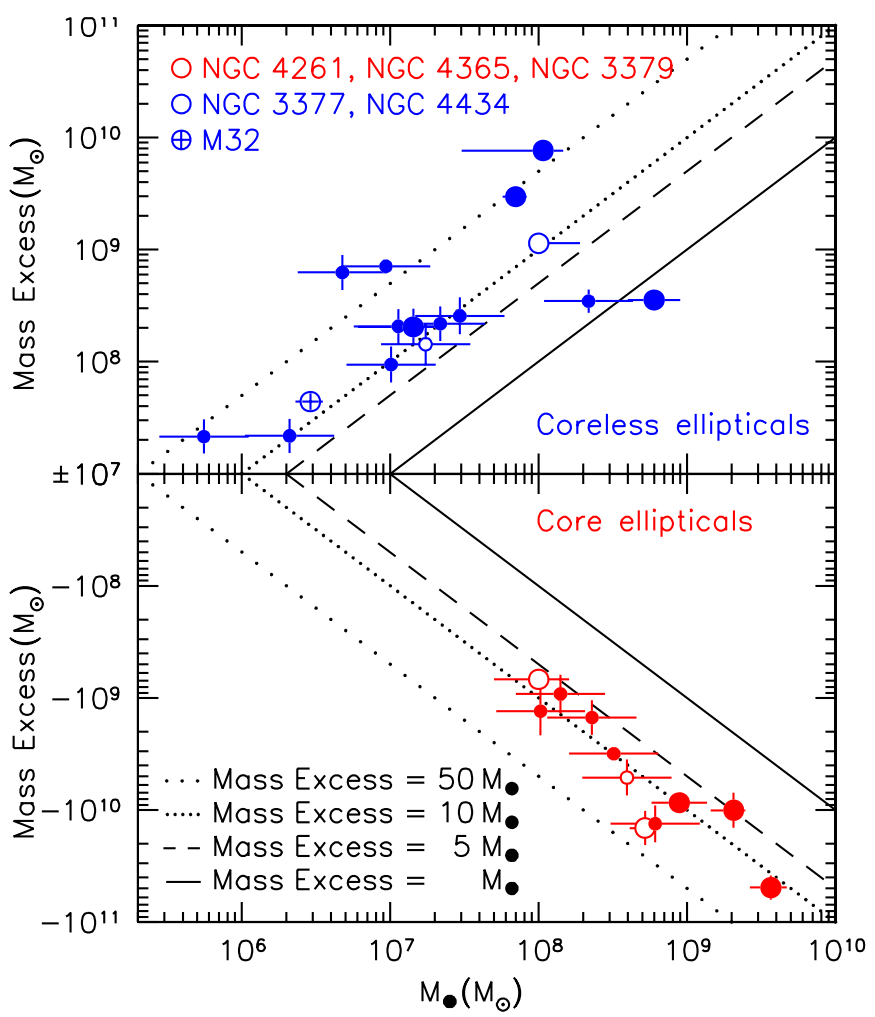

Figure 1. Stellar mass missing in cores (lower panel) or extra in coreless galaxies (upper panel) as a function of BH mass. Large and small symbols are for galaxies with and without dynamical $\mathrm{BH}$ detections. The sample is all known ellipticals in the Virgo cluster plus non-members denoted by open symbols. From Kormendy et al. (2008) with NGC 3379 added $\left(\log M_{\bullet}=8.0\right)$.

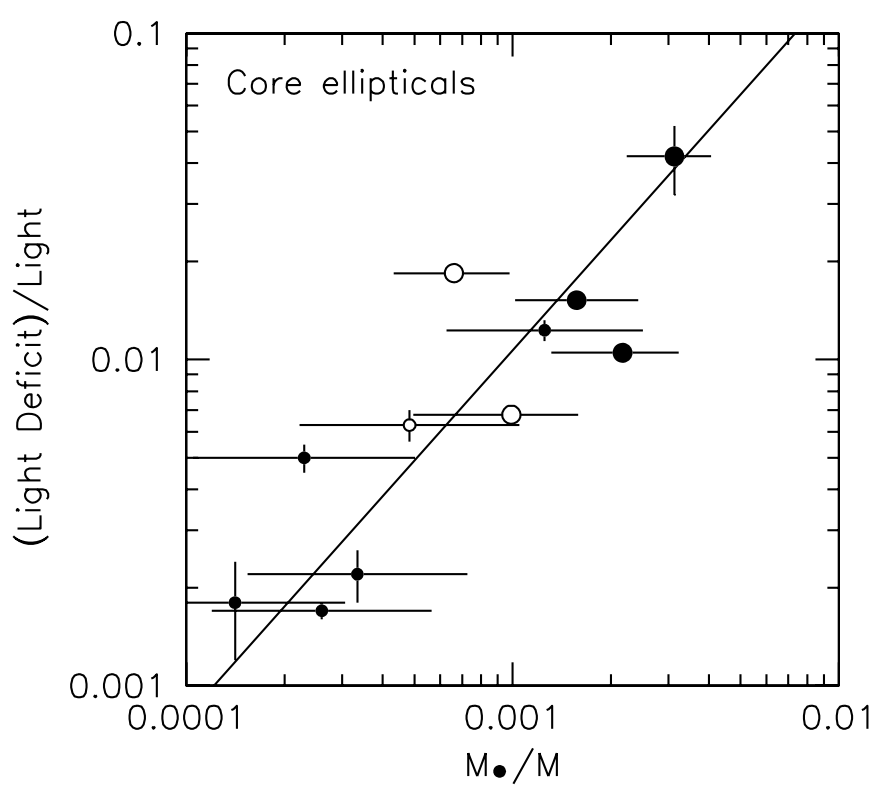

Figure 2. Fraction of the total $V$-band luminosity "missing" in cores vs. the ratio of $\mathrm{BH}$ mass to the total stellar mass of the galaxy. The sample is as in Figure 1 and Table 1. Large and small symbols denote galaxies with and without dynamical $\mathrm{BH}$ detections. The galaxies with $\mathrm{BH}$ detections are (top to bottom) M87 (Macchetto et al. 1997), NGC 4261 (Ferrarese et al. 1996), NGC 4374 (Bower et al. 1998), NGC 4649 (Gebhardt et al. 2003), and NGC 3379 (Gebhardt et al. 2000a). The line is Equation (1).

with rms scatter $=0.23$ in $\log L_{V \text {,def }} / L_{V}$ and 0.23 in $\log M_{\bullet} / M$. The latter is formally less than the error \pm 0.3 in $\log M_{\bullet}$ adopted

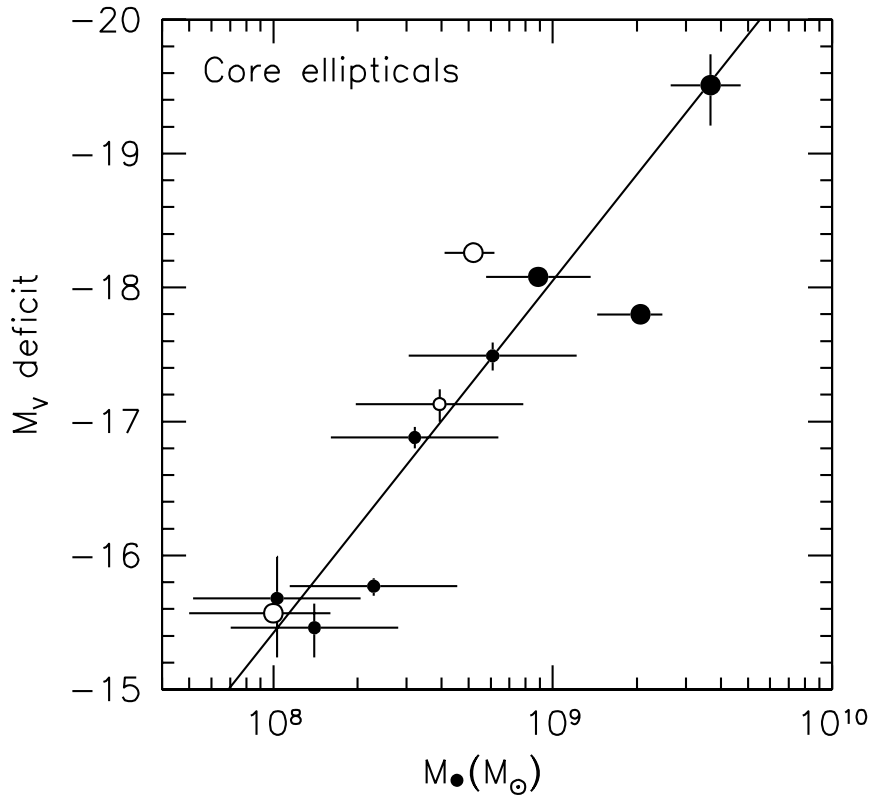

Figure 3. Absolute magnitude of the light that is missing in cores vs. BH mass The sample and symbols are as in Figure 2. The line is Equation (2).

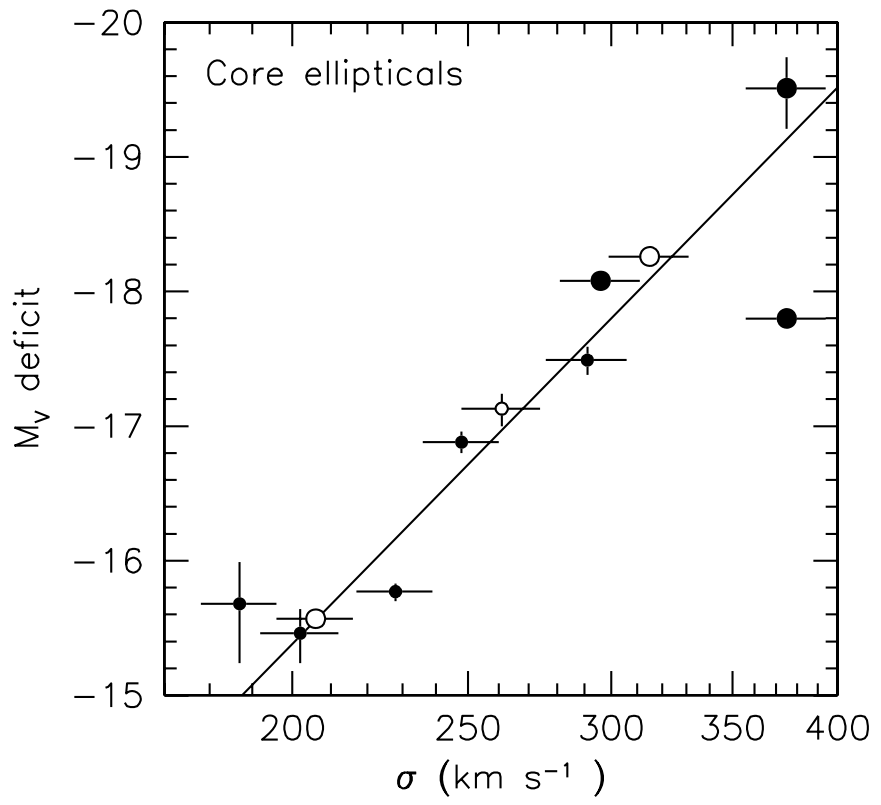

Figure 4. Absolute magnitude of the light missing in cores vs. galaxy velocity dispersion averaged inside a slit aperture whose length is approximately twice the half-light radius (Table 1).

by Tremaine et al. (2002). The fraction of the light that is missing in cores may be as good a predictor of $M_{\bullet}$ as is $\sigma$.

The canonical mean mass fraction in $\mathrm{BHs}$ is $M_{\bullet} / M \simeq 0.0013$ (Merritt \& Ferrarese 2001; Kormendy \& Gebhardt 2001). The five $\mathrm{BH}$ detections in Figure 2 are consistent with this value. But if velocity dispersions reliably tell us $\mathrm{BH}$ masses, then there is significant scatter in $M_{\bullet} / M$ toward lower values. Our observation that these lower $M_{\bullet}$ values are correlated with lower $L_{\text {def }}$ values adds confidence to our conclusion that $M_{\bullet} / M$ has significant cosmic scatter. The total range of $\log M_{\bullet} / M$ values found by Merritt \& Ferrarese (2001) and by Kormendy \& Gebhardt (2001) is more than \pm 1 around the mean. 
Figure 3 shows the absolute magnitude of the light that is missing in cores versus $\mathrm{BH}$ mass. It is analogous to previous derivations (see references in Section 1) of the correlations of mass deficits with $\mathrm{BH}$ masses, but $M_{V \text {, def }}$ does not involve the uncertainty of mass-to-light ratios.

The correlation in Figure 3 is remarkably good. The fit is

$$
M_{V, \text { def }}=(-18.05 \pm 0.22)-(2.63 \pm 0.42) \log \left(\frac{M_{\bullet}}{10^{9} M_{\odot}}\right)
$$

with rms scatter $=0.52$ in $M_{V \text {,def }}$ and 0.20 in $\log M_{\bullet}$. The latter is less than the error \pm 0.3 in $\log M_{\bullet}$ taken from Tremaine et al. (2002). Again, core light deficit may be as good a predictor of $M_{\bullet}$ as is $\sigma$. Figure 3 spans the range of dynamically measured $\mathrm{BH}$ masses observed to date in ellipticals that have cores.

For convenience, we can rewrite Equation (2) as

$$
\frac{M_{\bullet}}{10^{8} M_{\odot}}=\left(0.81_{-0.15}^{+0.18}\right)\left(\frac{L_{V, \text { def }}}{10^{8} L_{V \odot}}\right)^{0.95_{-0.13}^{+0.18}}
$$

Remarkably, the typical $M / L_{V} \sim 8$ almost cancels the factor of 10 ratio between $L_{V \text {, def }} / L_{V}$ and $M_{\bullet} / M$ in Equation (1). Within the errors, $M_{\bullet} \simeq L_{V \text {, def }}$, where both are measured in solar units.

We emphasize that the $M_{V \text {,def }}$ measurements in Figure 3 are independent of $M_{\bullet}$ except for the indirect dependence of $M_{\bullet}$ on the light distribution through the dynamical modeling. It is interesting that the scatter in Figure 3, which mainly involves central galaxy quantities, is marginally smaller than the scatter in Figure 2, which also involves the total galaxy luminosity.

\section{LIGHT DEFICIT AND GALAXY VELOCITY DISPERSION}

The observation that $M_{\bullet}$ correlates closely with both $\sigma$ and $M_{V \text {,def }}$ hints that there may be good correlation between $M_{V \text {,def }}$ and velocity dispersion. Figure 4 shows that this correlation is, in fact, excellent. Moreover, it is a correlation between pure observables, with no dynamical modeling involved.

The correlation in Figure 4 is

$$
M_{V, \mathrm{def}}=(-16.71 \pm 0.18)-(13.73 \pm 1.92) \log \left(\frac{\sigma}{250 \mathrm{~km} \mathrm{~s}^{-1}}\right)
$$

$$
\frac{L_{V, \text { def }}}{10^{8} L_{V \odot}}=\left(4.10_{-0.63}^{+0.75}\right)\left(\frac{\sigma}{250 \mathrm{~km} \mathrm{~s}^{-1}}\right)^{5.49 \pm 0.77} .
$$

The rms scatter is 0.55 in $M_{V \text {,def }}, 0.22$ in $\log L_{V \text {,def }}$, and 0.0401 in $\log \sigma$. Tremaine et al. (2002) estimate that the intrinsic dispersion in $M_{\bullet}$ at constant $\sigma$ is $0.23 \pm 0.05$ dex. Our observed scatter in $L_{V \text {,def }}$ is essentially the same and includes measurement errors. For our sample, $\sigma$ predicts $L_{V \text {, def }}$ slightly more accurately than it predicts $M_{\bullet}$.

Figures 2-4 are remarkably good correlations, considering the difficulty of the $M_{V \text {,def }}$ and $M_{\bullet}$ measurements. The care that we took with the KFCB photometry helps. But there was never a guarantee that the intrinsic scatter in such correlations would be small. It is especially surprising because only core galaxies participate. Extra light galaxies contain BHs too (Figure 1), but extra light shows little correlation with $M_{\bullet}$. KFCB suggest that core Es are made in dry mergers whereas coreless Es are made in wet mergers in which central starbursts build the extra light. Then the amount of extra light is controlled mainly by the gas fraction in the progenitors and the properties of the starburst and only secondarily by the energy feedback from young stars, supernovae, and nuclear activity. We regard Figures $1-4$ as further evidence for this explanation of the E-E dichotomy.

In core galaxies, the excellent mutual correlations between light deficit, velocity dispersion, and $\mathrm{BH}$ mass-with observed scatter roughly consistent with measurement errors-imply that cores and BHs are closely related. We interpret them as a "smoking gun" in favor of the idea that cores are scoured by BHs. Core formation was surprisingly regular, given the probably heterogeneous merger histories of elliptical galaxies.

Our "bottom line" conclusion is that, for the present galaxy sample, $L_{V \text {,def }}$ and $\sigma$ provide two independent and comparably accurate predictors of $\mathrm{BH}$ mass.

It is a pleasure to thank David Fisher and Mark Cornell for an enjoyable collaboration on KFCB. J.K. thanks Milos Milosavljević for helpful discussions on $\mathrm{BH}$ scouring. We made extensive use of the NASA/IPAC Extragalactic Database (NED), which is operated by Caltech and JPL under contract with NASA, of the HyperLeda database http://leda.univ-lyon1.fr and of NASA's Astrophysics Data System bibliographic services. This work was supported by the National Science Foundation under grant AST-0607490.

\section{REFERENCES}

Bender, R. 1988, A\&A, 193, L7

Bender, R., Saglia, R. P., \& Gerhard, O. E. 1994, MNRAS, 269, 785

Bender, R., Surma, P., Döbereiner, S., Möllenhoff, C., \& Madejsky, R. 1989, A\&A, 217, 35

Bower, G. A., et al. 1998, ApJ, 492, L111

Boylan-Kolchin, M., Ma, C.-P., \& Quataert, E. 2004, ApJ, 613, L37

Caon, N., Capaccioli, M., \& D’Onofrio, M. 1993, MNRAS, 265, 1013

Cappellari, M., et al. 2006, MNRAS, 366, 1126

Ebisuzaki, T., Makino, J., \& Okamura, S. K. 1991, Nature, 354, 212

Faber, S. M., et al. 1997, AJ, 114, 1771

Ferrarese, L., Ford, H. C., \& Jaffe, W. 1996, ApJ, 470, 444

Ferrarese, L., \& Merritt, D. 2000, ApJ, 539, L9

Ferrarese, L., et al. 2006, ApJS, 164, 334

Gebhardt, K., et al. 1996, AJ, 112, 105

Gebhardt, K., et al. 2000a, AJ, 119, 1157

Gebhardt, K., et al. 2000b, ApJ, 539, L13

Gebhardt, K., et al. 2003, ApJ, 583, 92

Graham, A. W. 2004, ApJ, 613, L33

Gualandris, A., \& Merritt, D. 2008, ApJ, 678, 780

Ho, L. C. Ed. 2004, Carnegie Observatories Astrophysics Series Vol. 1, Coevolution of Black Holes and Galaxies, (Cambridge: Cambridge Univ. Press)

Hopkins, P. F., Cox, T. J., Dutta, S. N., Hernquist, L., Kormendy, J., \& Lauer, T. R. 2008, ApJ, in press (arXiv:0805.3533)

Kormendy, J. 1985, ApJ, 295, 73

Kormendy, J. 1987, in IAU Symp. 127, Structure and Dynamics of Elliptical Galaxies, ed. T. de Zeeuw (Dordrecht: Reidel), 17

Kormendy, J. 1993, in The Nearest Active Galaxies, ed. J. Beckman, L. Colina, \& H. Netzer (Madrid: Consejo Superior de Investigaciones Científicas), 197

Kormendy, J. 1999, in Galaxy Dynamics: A Rutgers Symposium, ed. D. Merritt, J. A. Sellwood, \& M. Valluri (San Francisco, CA: ASP), 124

Kormendy, J., \& Bender, R. 1996, ApJ, 464, L119

Kormendy, J., Fisher, D. B., Cornell, M. E., \& Bender, R. 2008, ApJS, in press (arXiv:0810.1681) (KFCB)

Kormendy, J., \& Gebhardt, K. 2001, in 20th Texas Symposium on Relativistic Astrophysics, ed. J. C. Wheeler \& H. Martel (New York: AIP), 363

Kormendy, J., \& Richstone, D. 1995, ARA\&A, 33, 581

Kormendy, J., et al. 1994, in ESO/OHP Workshop on Dwarf Galaxies, ed. G. Meylan \& P. Prugniel (Garching: ESO), 147

Lauer, T. R. 1985, ApJ, 292, 104

Lauer, T. R., et al. 1995, AJ, 110, 2622

Lauer, T. R., et al. 2005, AJ, 129, 2138

Lauer, T. R., et al. 2007, ApJ, 664, 226 
Macchetto, F., Marconi, A., Axon, D. J., Capetti, A., Sparks, W., \& Crane, P. 1997, ApJ, 489, 579

Magorrian, J., et al. 1998, AJ, 115, 2285

Mei, S., et al. 2007, ApJ, 655, 144

Merritt, D. 2006, ApJ, 648, 976

Merritt, D., \& Ferrarese, L. 2001, MNRAS, 320, L30

Merritt, D., Mikkola, S., \& Szell, A. 2007, ApJ, 671, 53

Merritt, D., Milosavljević, M., Favata, M., Hughes, S. A., \& Holz, D. E. 2004, ApJ, 607, L9

Mihos, J. C., \& Hernquist, L. 1994, ApJ, 437, L47

Milosavljević, M., \& Merritt, D. 2001, ApJ, 563, 34
Milosavljević, M., Merritt, D., Rest, A., \& van den Bosch, F. C. 2002, MNRAS, 331, L51

Nieto, J.-L., Bender, R., \& Surma, P. 1991, A\&A, 244, L37

Ravindranath, S., Ho, L. C., \& Filippenko, A. V. 2002, ApJ, 566, 801

Ravindranath, S., Ho, L. C., Peng, C. Y., Filippenko, A. V., \& Sargent, W. L. W. 2001, AJ, 122, 653

Rest, A., et al. 2001, AJ, 121, 2431

Sérsic, J. L. 1968, Atlas de Galaxias Australes, (Cordoba: Observatorio Astronomico, Universidad de Cordoba)

Tonry, J. L., et al. 2001, ApJ, 546, 681

Tremaine, S., et al. 2002, ApJ, 574, 740 